\title{
La liaison fructueuse: Laboratory and emergency medicine
}

\author{
Giuseppe Lippi, ${ }^{1}$ Gianfranco Cervellin ${ }^{2}$ \\ ${ }^{1}$ Section of Clinical Biochemistry, University Hospital of Verona, Verona; ${ }^{2}$ Emergency Department, \\ University Hospital of Parma, Parma, Italy
}

\begin{abstract}
Laboratory diagnostics is traditionally defined as a specific medical branch dedicated to produce clinically useful information through quantitative or qualitative analysis of analytes in biological fluids. ${ }^{1}$ The effective contribution that in vitro diagnostic testing convey to both the diagnostic reasoning and the managed care is still a matter of discussion. Earlier published claims supported the assumption that the medical laboratory may intervene in up to $70 \%$ of clinical decisions, ${ }^{2}$ whilst no evidence-based information has been brought in support of this statement. Although we would all agree that most clinical decisions cannot be taken without laboratory information, it is also undeniable that some care pathways rely more strongly on diagnostic testing than others. Could you imagine investigating chest pain without measuring cardiac troponins? Or diagnosing diabetes without measuring plasma glucose or hemoglobin A1c ? ${ }^{3}$ These are just two paradigmatic examples of how laboratory testing not only has considerably improved (and guided) the diagnostic reasoning, but that it has also contributed to revolutionize the current definition and management of many human diseases.
\end{abstract}

In 1986, the American College of Emergency Physicians (ACEP) originally defined Emergency Medicine as the medical specialty dedicated to the diagnosis and treatment of unforeseen illness or injury. The aforementioned definition specifies that the practice of emergency medicine includes the initial evaluation, diagnosis, treatment, coordination of care among multiple providers, and disposition of any patient requiring expeditious

Correspondence: Giuseppe Lippi, Section of Clinical Biochemistry, University Hospital of Verona, Piazzale L.A. Scuro, 37134 Verona, Italy.

E-mail: giuseppe.lippi@univr.it

Key words: Laboratory medicine; Emergency medicine; Diagnosis; Testing.

Contributions: the authors contributed equally.

Conflict of interest: the authors declare no potential conflict of interest.

Funding: none.

Received for publication: 29 May 2019.

Accepted for publication: 14 June 2019.

This work is licensed under a Creative Commons Attribution 4.0 License (by-nc 4.0).

(C) Copyright: the Author(s), 2019

Licensee PAGEPress, Italy

Emergency Care Journal 2019; 15:8344

doi:10.4081/ecj.2019.8344 medical, surgical, or psychiatric care, and that Emergency Medicine is not defined by location, but may be practiced in a variety of settings including hospital-based and freestanding emergency departments (EDS), urgent care clinics, observation medicine units, emergency medical response vehicles, at disaster sites, or via telemedicine. ${ }^{4}$ This definition was subsequently minimally revised and reaffirmed in 1994, 1998, 2001, 2008 and 2015..$^{5}$

The leading paradigms of emergency medicine encompass rapid and accurate diagnoses, followed by appropriate treatments. This would actually require a substantial adaptation of subsidiary diagnostic sciences, such as laboratory medicine, which shall modify their organization to provide rapid tests, characterized by high diagnostic accuracy, for enabling emergency physicians to make early rule out or diagnoses. ${ }^{6}$ Fulfilling these essential requirements can only be accomplished through a strict partnership between laboratory professionals and emergency physicians, who should actively cooperate and collaborate to design the urgent test menus according to their characteristic nature. Essentially, this translates into the clear-cut concepts that: i) emergency physicians shall pose diagnostic questions to the laboratory, and the laboratory - in turn - should be embarked into a constructive effort to rearrange its organization to fulfill these needs whilst, ii) laboratory professionals shall loyally discuss with emergency physicians the locally availability of tests and, altogether, they should define a reliable panel of analyses that can be ordered in the emergency room. Notably, clinical needs shall always be evaluated for cost-effectiveness, since distracting resources for obsolete, redundant or clinically questionable tests will generate adverse consequences for the entire healthcare system. An emblematic example is that described by Almazini et al. in the previous issue of the journal, ${ }^{7}$ who showed that routine measurement of natriuretic peptides at hospital admission and discharge was associated with increased medical expenditures, whilst favorable impacts on clinical outcomes could be observed.

The establishment of a vicious (virtuous) circle between emergency and laboratory medicine seems hence essential for optimizing the use of human and technical resources in both environments, but is also advisable for obtaining the best possible outcomes for the patients (Figure 1). Two Italian scientific societies of emergency medicine (Academy of Emergency Medicine and Care, AcEMC) and laboratory medicine (Italian Society of Clinical Biochemistry and Clinical Molecular Biology, SIBioC) have been forerunner in pursuing this approach, and many important targets have already been achieved by publishing consensus documents on many important issues, such as the panels of urgent tests, ${ }^{8,9}$ the diagnostics of myocardial infarction, ${ }^{10}$ sepsis ${ }^{11}$ and venous thromboembolism. ${ }^{12}$ Nevertheless, some major hurdles remain, which may ultimately challenge the effective liaison between emergency and laboratory medicine.

Defensive medicine is indeed the first of such tasks. The emergency settings are especially vulnerable to malpractice and liability, ${ }^{13}$ thus predisposing to inappropriate usage or overuse of laboratory resources. ${ }^{14}$ This inappropriate and unwarranted practice has many inherent drawbacks, such as the enhanced risk of generating 
false positive results, disrupting laboratory workflow, increasing complexity in information management, prolonging the turnaround time and wasting both human and economic healthcare resources. On the other hand, inappropriateness also encompasses underuse of otherwise necessary or useful tests and, even in such case, the clinical consequence of a missed or delayed diagnosis can be detrimental. ${ }^{15}$

A timely laboratory response to an urgent clinical need is another important problem, decidedly dependent on the local hospital organization. Due to the ongoing reorganization of laboratory services, mostly driven by economic rather than clinically-centered goals, ${ }^{16}$ many clinical laboratories have been shut down, outsourced or relocated far from the emergency departments, thus catalyzing many conceptual, technical and practical caveats in urgent testing. ${ }^{17}$ Although the efforts made for counteracting this essentially politically-driven strategy have been virtually ineffective in recent times, some pragmatic solutions could be identified, such as widespread implementation of pneumatic tube systems, sample transportation by innovative devices (e.g., drones) $)^{18}$ or point-ofcare testing, as thoughtfully discussed by Carraro. ${ }^{19}$

A third common obstacle for an effective partnership between emergency and laboratory medicine is the mutual lack of sufficient knowledge of each other work. The basic questions are: i) how much do laboratory professionals know about the critical role that emergency physicians play in rapidly managing critical patients within an overcrowded environment? and ii) how much do emergency physicians know about the critical role that laboratory professionals play in overseeing the constantly expanding volume and complexity of in vitro diagnostic testing? Some viable solutions can also be found to overcome this problem, mostly relying on the elemental concept of enhanced or improved mutual communication, which develops through major involvement in interdisciplinary teams and care pathways, organization of joint meetings and conferences, as well as in establishment of multidisciplinary societies or organizations (such as AcEMC) where emergency physicians and laboratory professionals can confront and discuss clinical, practical, technical and even political problems. The collection of spuriously hemolyzed blood samples is a brilliant example. Emergency physicians, and especially emergency nurses, often perceive this problem as a laboratory failure, whilst an unsuitable specimens is more frequently caused by preanalytical issues

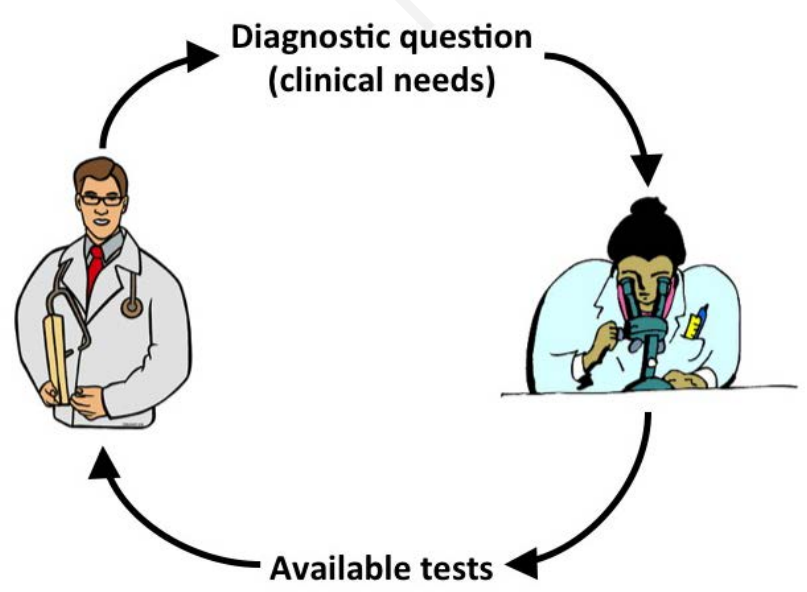

Figure 1. La liaison fructueuse between laboratory and emergency medicine. ascribable to an inappropriate blood drawing technique. Unfortunately, hemolyzed samples may be associated with a substantial burden of diagnostic errors when not timely identified, and also with a consistent diagnostic and therapeutic delay. ${ }^{20,21}$ Nevertheless, reinforced and proactive liaison between the emergency department and laboratory service was proven effective to consistently reduce the frequency of hemolyzed samples, thus improving both clinical outcomes (i.e., more timely diagnosis and treatment) and organizational issues (lower costs, less overcrowding). ${ }^{22,23}$ Another interesting instance on how laboratory testing can help organization together with clinical practice in the emergency room is suggested by another article published in this issue of the Journal, ${ }^{24}$ according to which the temporal analysis of alcohol tests requested in the ED may generate valuable information for proactively addressing organizational issues in both environments (i.e., the risks of ED overcrowding and lack of staff or reagents in the laboratory).

A fourth and final aspect contributing to disrupt the liaison between emergency and laboratory medicine is the occasionally limited understanding of the real significance and clinical implication of some laboratory tests. False myths and misconceptions are still particularly frequent in clinical practice, as thoughtfully highlighted elsewhere. ${ }^{25}$ Some emblematic examples include increased cardiac troponin values always reflecting acute myocardial infarction (whilst enhanced concentrations are commonplace in a kaleidoscope of non-ischemic myocardial injuries), prolonged values of activated partial thromboplastin time are always associated with bleeding (whilst they can also be due to the innocent coagulation factor XII deficiency), abnormal D-dimer concentrations are always diagnostic of venous thromboembolism (whilst D-dimer value is also increased in a vast array of non-thrombotic disorders). ${ }^{26}$ In such case, a reinforced laboratory stewardship seems essential for improving physicians' knowledge on the many clinical implications of diagnostic testing.

In conclusion, the reinforcement of laboratory and emergency medicine liaison seems now an unavoidable strategy for making urgent healthcare management a more efficient and sustainable enterprise.

\section{References}

1. Plebani M, Lippi G. Uncertainty, quality, safety and accreditation in laboratory medicine. J Lab Precis Med 2017;2:80.

2. Hallworth MJ. That ' $70 \%$ ' claim again. Ann Clin Biochem 2018;55:517-8

3. Lippi G. The irreplaceable value of laboratory diagnostics: four recent tests that have revolutionized clinical practice. eJIFCC 2019;30:7-13.

4. American College of Emergency Physicians. Definition of emergency medicine and the emergency physician. Ann Emerg Med 1986;15:1240-1.

5. American College of Emergency Physicians. Definition of emergency medicine. Ann Emerg Med 2016;68:142-3.

6. Lippi G, Guidi GC. The power of negative thinking. Am J Emerg Med 2008;26:373-4.

7. Almazini P, Siswanto B, Meyer M, et al. An health economic evaluation of using $\mathrm{N}$-terminal pro brain natriuretic peptide for the management of acute heart failure: A pilot study in an Indonesian tertiary referral hospital. Emerg Care J 2019;15: 7919.

8. Lippi G, Panteghini M, Bernardini S, et al. Laboratory testing 
in the emergency department: an Italian Society of Clinical Biochemistry and Clinical Molecular Biology (SIBioC) and Academy of Emergency Medicine and Care (AcEMC) consensus report. Emerg Care J 2017;13:6600.

9. Lippi G, Balboni F, Bonfanti L, et al. Laboratory medicine and emergency medicine: an essential partnership. Biochim Clin 2018;42:335-42.

10. Casagranda I, Cavazza M, Clerico A, et al. Proposal for the use in emergency departments of cardiac troponins measured with the latest generation methods in patients with suspected acute coronary syndrome without persistent ST-segment elevation. Clin Chem Lab Med 2013;51:1727-37.

11. Giannakopoulos K, Hoffmann U, Ansari U, et al. The use of biomarkers in sepsis: a systematic review. Curr Pharm Biotechnol 2017;18:499-507.

12. Lippi G, Cervellin G, Casagranda I, et al. D-dimer testing for suspected venous thromboembolism in the emergency department. Consensus document of AcEMC, CISMEL, SIBioC, and SIMeL. Clin Chem Lab Med 2014;52:621-8.

13. Cervellin G, Cavazza M. Defensive medicine in the emergency department. The clinicians' perspective. Emerg Care J 2016;12:5615.

14. Montagnana M, Lippi G. The risks of defensive (emergency) medicine. The laboratory perspective. Emerg Care J 2016;12:5581.

15. Lippi G, Bovo C, Ciaccio M. Inappropriateness in laboratory medicine: an elephant in the room? Ann Transl Med 2017;5:82.

16. Lippi G, Bassi A, Bovo C. The future of laboratory medicine in the era of precision medicine. J Lab Precis Med 2016;1:7.
17. Plebani M, Laposata M, Lippi G. A manifesto for the future of laboratory medicine professionals. Clin Chim Acta 2019;489:49-52.

18. Lippi G, Mattiuzzi C. Biological samples transportation by drones: ready for prime time? Ann Transl Med 2016;4:92.

19. Carraro P. The point-of-care testing in the emergency department. Emerg Care J 2019;15:8019.

20. Lippi G, Plebani M, Di Somma S, et al. Hemolyzed specimens: a major challenge for emergency departments and clinical laboratories. Crit Rev Clin Lab Sci 2011;48:143-53.

21. Lippi G, Cervellin G, Mattiuzzi C. Critical review and metaanalysis of spurious hemolysis in blood samples collected from intravenous catheters. Biochem Med (Zagreb) 2013;23:193200.

22. Lippi G, Bonelli P, Bonfanti L, et al. The use of S-Monovette is effective to reduce the burden of hemolysis in a large urban emergency department. Biochem Med (Zagreb) 2015;25:6972.

23. Duhalde H, Skogö J, Karlsson M. Point-of-care hemolysis detection in blood gas specimens directly at the emergency department. Scand J Clin Lab Invest 2019;8:1-5.

24. Lippi G, Ferrari A, Bovo C, Cervellin G. Hospital admissions for alcohol-related problems in concomitance with weekends, New Year's Eve and Christmas Eve: Myth or reality? Emerg Care J 2019;15:8024.

25. Lippi G, Cervellin G, Plebani M. The ten commandments of laboratory testing for emergency physicians. Clin Chem Lab Med 2014;52:183-7.

26. Lippi G, Plebani M. False myths and legends in laboratory diagnostics. Clin Chem Lab Med 2013;51:2087-97. 